\title{
New paradigms on siRNA local application
}

\author{
Meng Pan ${ }^{1,2, \#}$, Jinwen $\mathrm{Ni}^{1, \#}$, Huiming $\mathrm{He}^{2}$, Shan Gao ${ }^{3} \mathcal{E}$ Xiaohong Duan ${ }^{1, *}$ \\ ${ }^{1}$ State Key Laboratory of Military Stomatology, Department of Oral Biology, School of Stomatology, the Fourth Military Medical University, \\ Xi'an 710032, Shaanxi, People's Republic of China, ${ }^{2}$ State Key Laboratory of Military Stomatology, Department of Prosthodontics, School \\ of Stomatology, the Fourth Military Medical University, Xi'an 710032, China, ${ }^{3}$ School of Stomatology, Central South University, Changsha \\ 410078, Hunan, People's Republic of China
}

Small interfering RNA (siRNA) functions through pairing with specific mRNA sequences and results in the mRNA's degradation. It is a potential therapeutic approach for many diseases caused by altered gene expression. The delivery of siRNA is still a major problem due to its rapid degradation in the circulation. Various strategies have been proposed to help with the cellular uptake of siRNA and short or small hairpin RNA (shRNA). Here, we reviewed recently published data regarding local applications of siRNA. Compared with systemic delivery methods, local delivery of siRNA/shRNA has many advantages, such as targeting the specific tissues or organs, mimicking a gene knockout effect, or developing certain diseases models. The eye, brain, and tumor tissues are 'hot' target tissues/organs for local siRNA delivery. The siRNA can be delivered locally, in naked form, with chemical modifications, or in formulations with viral or non-viral vectors, such as liposomes and nanoparticles. This review provides a comprehensive overview of RNAi local administration and potential future applications in clinical treatment. [BMB Reports 2015; 48(3): 147-152]

\section{INTRODUCTION}

The RNA interference (RNAi) phenomenon is a gene-silencing process targeting and degrading specific mRNA molecules by base paring, consequently suppressing protein expression levels (1). The mechanism of the highly conserved interference process was first found in plants and then in other eukaryotes in subsequent studies (2). RNAi starts from the cytoplasm, where a long double-stranded RNA (dsRNA) molecule or shRNA is cleaved by the enzyme Dicer into a $\sim 20$ bp short dsRNA mol-

*Corresponding author. Tel: +86-29-84776169; Fax: +86-2984776169; E-mail: xhduan@fmmu.edu.cn

${ }^{\text {\#} C o-f i r s t ~ a u t h o r, ~ c o n t r i b u t e d ~ e q u a l l y ~ t o ~ t h i s ~ w o r k . ~}$

http://dx.doi.org/10.5483/BMBRep.2015.48.3.089

Received 29 April 2014, Revised 26 May 2014, Accepted 31 July 2014

Keywords: In vivo, Local injection, shRNA, siRNA ecule, which is called siRNA. During the in vivo delivery process, siRNA faces big challenges to reach target cells (S1). For example, ribonucleases may limit the half-life time of a naked siRNA molecule in the circulation to less than $1 \mathrm{~min}$ (S2). Moreover, the negative charge and large size of a naked siRNA make it difficult to pass through the plasma membrane of a target cell (3). To avoid these problems, in vivo local delivery of siRNA has been advocated over the past decade. Here, we review recent findings on the local injection of siRNA, which may be regarded as an alternative therapeutic approach for future clinical treatments, especially in specific organs or for certain diseases.

\section{SIRNA FORMULATIONS}

To overcome the challenges of siRNA/shRNA delivery, various of strategies have been used to increase RNAi efficiency, including viral vector-based delivery, and non-viral liposomes and nanoparticles, as well as naked siRNA with chemical modifications.

\section{Viral vector-mediated siRNA delivery}

Several viral vector-mediated siRNA delivery systems, such as lentivirus vectors (LV), adenovirus-associated vectors (AAV), adenovirus vectors (AV), and retrovirus vectors (RV) have been developed and assessed (S3) (Table 1). Given their relatively low immunogenicity, general safety, and high transfection efficiencies, adenovirus and lentivirus vectors are the most widely used viral vectors today. Because of the effective, sustained, and stable properties of transfection, virus-mediated RNAi is a powerful tool in inhibiting the expression of specific genes (S4). The approach has been applied in tumor therapy to arrest the cell cycle and prevent tumor growth, and it has also been discussed in treating amyotrophic lateral sclerosis (ALS), Huntington's disease (HD) and Alzheimer's disease in recent years.

However, virus-mediated siRNA may have potential safety problems, and may lead to severe complications (S5), such as allergic, immune, and inflammatory responses. More efforts are still needed for the use of virus-mediated siRNA delivery in future therapeutics (S6). 
Table 1. Comparison of viral vector delivery system

\begin{tabular}{|c|c|c|c|}
\hline Categories & Advantages & Disadvantages & Immune stimulation \\
\hline Adenovirus-associated vectors (AAV) & Long term effect; non-pathogenic & Small packaging size & Very low \\
\hline Lentivirus vectors (LV) & $\begin{array}{l}\text { Long-term effect in most tissues. } \\
\text { Stable and effective. }\end{array}$ & Safety problem. May induce neoplasia. & Low \\
\hline Retrovirus vectors (RV) & More stable & Safety problem. Potential neoplasia problem. & Moderate \\
\hline Adenovirus vectors (AV) & High efficiency and stability & Inflammatory and immune responses & High \\
\hline
\end{tabular}

Table 2. Advantages and disadvantages of non-viral vector delivery systems

\begin{tabular}{|c|c|c|}
\hline & Advantages & Disadvantages \\
\hline Naked siRNA & $\begin{array}{l}\text { Effective in certain organs. Lower toxicity and } \\
\text { inflammatory responses. Simple formulation. }\end{array}$ & Lack of stability. \\
\hline Chemically modified siRNA & $\begin{array}{l}\text { Easily formulated. Medium efficiency. Lower immune } \\
\text { response. }\end{array}$ & $\begin{array}{l}\text { Impaired bioactivity. High Cost. Toxicity. } \\
\text { Inflammatory response. }\end{array}$ \\
\hline Liposome modified siRNA & $\begin{array}{l}\text { Stable, variable, flexible and multifunctional. Easy to } \\
\text { formulate. Biodegradable. No immune response and } \\
\text { non-toxic in neutral liposome. More effective in cationic } \\
\text { liposomes. }\end{array}$ & $\begin{array}{l}\text { Low specificity. Low efficiency in neutral lipids. } \\
\text { Inflammatory results with cationic lipids. }\end{array}$ \\
\hline Nanoparticles & $\begin{array}{l}\text { High stability. Decreased non-specific bio-distribution. } \\
\text { Induces non-specific endocytosis. }\end{array}$ & Cell toxicity \\
\hline
\end{tabular}

\section{Non-viral vector-mediated siRNA delivery (Table 2)}

Naked siRNA: A naked siRNA molecule is prone to be degraded by enzymes in the body environment, especially in the blood stream. However, in certain organs, such as the eye, brain, ear, skin and heart, local injection of naked siRNA has been reported to achieve the goal of gene silencing (S7-S11).

Chemically modified siRNA: To improve the in vivo stability and specificity, reduce toxicity, increase cellular uptake, and avoid immune and off-target responses, chemical and structural modifications of siRNA molecules are used widely (S12), without decreasing the silencing efficiency (S13). These includes ribose modifications, base modifications, and modifications within the phosphate backbone (S14-S15).

A potent single-stranded siRNA (ss-siRNA) requires a $5^{\prime}$ phosphate to achieve activity in correlation with Argonaute $\left(\mathrm{AGO}_{2}\right)$ instead of a liposome formulation. This method offers an alternative RNAi mechanism, but is still far from application in RNAi therapeutics (4).

Liposome-formulated siRNA: Lipid-based cationic liposomes are emerging as common non-viral gene delivery carriers. The structure of a liposome-siRNA nanoparticle consists of an internal aqueous compartment surrounded by an outer phospholipid bilayer (S16). Membrane fusion is the approach by which the liposome facilitates siRNA delivering into the target cell (S17). siRNA encapsulated within liposomes can be further modified with peptides, that may increase siRNA uptake by specific cell types and has been demonstrated to be less toxic (S18). Differing from liposomes, lipoplexes are formed with anionic siRNA combined with cationic lipids via electrostatic interactions (5). The properties of the formulation are similar to the crude siRNA lipoplexes to which anionic polymers have been added. Seven such formulations were summarized and demonstrated to be biodegradable and non-toxic with a variety of features (S19).

Nanoparticles: Nanoparticles are formed when cationic polymers interact with anionic siRNA by electrostatic interactions. There are various types of polymers today, such as chitosan, polyethylenimine $(\mathrm{PEI})$, dendrimers, cyclodextrin $(\mathrm{CD})$, and metallic core nanoparticles.

Chitosan is one polymer that has been widely studied. It is extracted from crustacean shells, so it is inexpensive and readily available. Chitosan also has excellent biocompatibility, biodegradability, adsorbability, and low toxicity and immunogenicity $(6, \mathrm{~S} 20)$. Poly (ethyleneimine) (PEI) is a synthetic polymer that can be linear or branched, and has a high percentage of free amine groups, which are positively charged. PEI-based nanocarriers can use PEI alone or be modified with PEG or other molecules to increase the stability of the dsRNA molecule and the circulation time of PEI-RNA particles, because PEI, a hydrophilic molecule, does not bind serum proteins. The drawback of adding PEG to PEI is that it will reduce the positive charge of the polymer, thus lessening its interaction with cell membranes, which leads to decreased uptake of the PEI/PEG particles (S6).

siRNA-formulated nanoparticles also show particular characteristics. When irradiated by UV light for 30s, photodegradable siRNA nanoparticles can be released (S21). siRNA within a 'multifunctional envelope-type nanodevice' (MEND) provided an alternative way in treating lung diseases via targeting the lung endothelium, which relies on the existence of a 
surface-modified GALA peptide (S22). PEG-CCP/CaP (polyethylene glycol block charge conversional polymer/calcium phosphate) hybrid micelle nanocarriers for siRNA delivery were used recently in the treatment of spontaneous pancreatic tumors with high effectiveness and safety (7).

\section{SIRNA APPLICATION MODES}

The siRNA can be delivered in two ways: systemic and local application. For systemic delivery, the most common forms are intravenous injection and intra-peritoneal injection (8, S23-S25). Systemic delivery can achieve the gene silencing goal far from the injection site. Compared with local injection, systemic delivery relies on higher injection doses, more dependence on carriers, and more extensive modifications of the siRNA (S26).

\section{'HOT' ORGANS FOR LOCAL INJECTION}

Various siRNA/shRNA formulations have been administered locally to different organs or tissues (e.g., eye, brain, tumor, dermis, muscle, ear, joint and heart) avoiding systemic issues. In most cases, certain forms of formulated siRNA are still required. Viral vector-mediated siRNA, such as adenovirusmediated siRNA have been reported in research on the eye (subretinal), brain (striatum, mediobasal hypothalamus), muscle (triceps surae muscle), and joints (intra-articulation). Lentivirus-formulated siRNAs have been used in the brain (hippocampus) and joints (intra-articulation). Naked siRNA has been injected locally into the eye (intraocular to retinal terminals in the superior colliculus, intravitreal to optic cup), brain (somatosensory cortex surface, striatum), ear (antero-inferior region of middle ear), and heart (apex of left ventricular). Chemically modified siRNAs have usually been used in the eye (intravitreal/periocular), brain (dorsal third ventricle/dopaminergic ventral midbrain, corpus callosum). Lipid/liposomemodified siRNAs have also been administered in the eye (subretinal, intravitreal to vitreous cavity). All siRNA formulations, including nanoparticles, have been used for intratumoral delivery.

\section{Eye}

The eye has been one of the most popular organs for siRNA local injection in recent years. Naked siRNA or formulated siRNAs can achieve the goal of specific gene silencing. To sum up recently published data about the local application of siRNA in the eyes, we found that lipid- or liposome-formulated siRNA were most commonly used (38\%), followed by naked siRNA (25\%). The local application of siRNA showed great advantages in exploring specific gene function, mimicking gene knockout effect, and developing certain diseases models (Supplementary Table 1).

Exploration of gene function in vivo: The first reported case to suppress gene expression in the synapse in vivo involved the subretinal administration of App (amyloid precursor pro- tein)-specific shRNA in rat retinal cells, which resulted in decreased App content in the superior colliculus and damaged synaptic activity. This application mimicked the possible pathogenic mechanism of App in Alzheimer's disease (AD) (S7).

The subretinal delivery of lipid-siRNA targeting complement factor $H$ (Cfh) in mice was reported to result in a knock-down of $C f h$ expression in the retinal pigmented epithelium and choroid, suggesting that $C f h$ plays an important role in the development of choroidal neovascularization (CNV) by regulating membrane attack complex formation (S27).

Other gene function studies involved liposome-formulated siRNAs in silencing hypoxia-inducible factor 1alpha (Hif1 $\alpha$ ) and vascular endothelial growth factor (Vegf) in murine retina (S28), and naked siRNA or shRNA of Hemeoxygenase (Ho)-2, respectively (S7).

Generation of disease models: The intravitreal delivery of specific chemically modified siRNA could reduce the expression of caspase-2, which in involved in retinal ganglion cell (RGC) apoptosis, caused by optic nerve injury. These data may provide a therapeutic method in treating ophthalmic diseases that involve RGC apoptosis (9). Glaucoma is characterized by high intraocular pressure (IOP) due to an imbalance in the aqueous humor, related to the $\beta 2$-adrenergic receptor (ADRB2). siRNA drug SYL040012 was designed against $A D R B 2$ and was administered in healthy volunteers. The results demonstrated that SYL040012 was well tolerated and effective in suppressing ADRB2 without side effects (10). Thus SYL040012 could be a potential way of treating glaucoma by inhibiting the production of aqueous humor (11).

\section{Brain}

Great efforts have been made regarding the local delivery of naked siRNA or formulated siRNA to the brain in recent years. Chemically modified siRNA was chosen in $30 \%$ of the published injection data. Viral vector-mediated shRNA delivery was also used frequently in brain disease research (Supplementary Table 2).

Generation of disease models: Because it is known that HD is caused by mutant huntingtin $(H t t)$, in a new technique, single-stranded siRNA (ss-siRNA) was infused intraventricularly to selectively silence the mutant $H t t$ allele in a mouse HD model. This may lead to better options for siRNA therapeutics in HD patients (12). Cholesterol-conjugated siRNA (cc-siRNA) targeting huntingtin $(H t t)$ was administered directly into the striatum in a HD mouse model. The Htt gene was silenced locally and diminished neuronal pathology was observed (13).

After injection of cc-siRNA targeted to the Cnpase gene in oligodendrocytes in the mouse corpus callosum, enhanced attenuation of CNPase was observed in the conjugate group versus the control group (S29).

Exploration of gene function in vivo: Injection of DOTAP-conjugated siRNA into the ventral tegmental/substantia nigra areas of the brain striatum in adult mice targeting the dopamine 
transporter (Dat) or tyrosine hydroxylase (Th), resulted in reduced mRNA levels in the striatum and subsequent behavioral changes (S30).

Microinjected siRNA targeted against Growth hormone-releasing hormone receptor (Ghrhr) into rat somatosensory cortex resulted in the suppression of Ghrhr expression, and influenced sleep, changing EEG delta waves, indicating that local administration of siRNA in vivo may be a useful approach to explore the mechanism of sleep (S9).

Mice injected locally with Cb1r siRNA and Cb1r knockout mice shared the same phenotypes, indicating that the CB1 receptor is involved in learning and CA3-CA1 synaptic plasticity (S31).

\section{Tumors}

Local injections of siRNA have been widely applied in the research of tumor therapy. Nanoparticles are the most frequently used siRNA formulations, accounting for $50 \%$. Up to $25 \%$ of lipid or liposome was chosen as the second most popular option of siRNA (Fig. 1) (S32-S45, 7, 14-18) (Supplementary Table 3).

Nanoparticle siRNA formulations: The following materials have been used to formulate siRNA: chitosan, PEI, polyelectrolyte complex (PEC), and PEG-CCP/CaP. With intratumoral delivery of siRNA and pegylated chitosan (CS)-poly-L-arginine (PLR) forming nanoparticles, PLR-grafted CS showed the highest reduction of function compared versus the control groups (S32). Selective knockdown of Stat3 expression by nanoparticles of siRNA-PEI modified with stearic acid (StA) was confirmed after intratumoral injection, and showed a more dramatic decrease in tumor volume than that of siRNA-PEI complexes (S33). PEC micelle-based Vegf siRNA was delivered intratumorally to lower Vegf levels in a mouse tumor model, resulting in suppressed tumor volume (S34).

Intravenous injection of PEG-CCP/CaP hybrid micelle-encapsulated siRNA resulted in the amelioration of spontaneously bioluminescent pancreatic tumors (7). When the athymic mouse was administered Aptamer-siRNA chimeras targeting prostate cancer cells, the tumor was suppressed markedly. The gene-silencing effect of Aptamer-siRNA chimeras was improved by adding two-nucleotide 3 '-overhangs and reducing formation of the duplex and the thermodynamic properties, as well as shortening the Aptamer portion of the chimeras (14). Two nucleolin Aptamer-siRNA chimeras (aptNCL-NRP1siR and aptNCL-SLUGsiR) were processed in lung cancer metastasis and decreased the expression of Nrp1RP1 and Slug, subsequently impairing the motility of the tumor cells, cancer growth, and invasiveness, without any apparent effect on normal cells. Similar results were obtained in a xenograft mouse model (15).

Lipid- or liposome-encapsulated siRNA: Cationic liposome combined with siRNA targeting 'receptor activator of nuclear factor kappa $B$ ligand' (RANKL) was injected intratumorally in an osteosarcoma model. Rankl production and tumor volume were reduced, which suggests a therapeutic approach for bone protection from tumor osteolysis and increased chemotherapeutic effect from osteosarcoma as a potential and promising tool (S35). Intratumoral injection of RFP specific siRNA (siRFP) and DG-based liposome complexes was reported to result in a reduction in RFP expression in the tumor tissues of mice (S36)

High-efficiency dicer-substrate short-interfering RNAs (DsiRNAs) in a lipid nanoparticle formulation were processed to shut off beta-catenin expression in a liver tumor model, indicating that DsiRNAs, may be a potential therapeutic method for treating traditionally undruggable cancers in the future (S37). Endothelial cells of tumor (TECs) are considered a key point in the pathological angiogenesis associated with tumor growth and development. Cyclo (Arg-Gly-Asp-D-Phe-Lys) (cRGD) was expressed highly in TECs. Cyclic RGD-modified liposomal-siRNA in vivo injection resulted in a reduction of target gene expression in TECs and significant inhibition in tumor growth (16). Intraductal injection of lipidoid nanoparticle-formulated siRNA targeting HoxA1 resulted in the suppression of mammary tumor growth in mice (17). Tumor-penetrating microparticles (TPM) improved the effects of lipid-modified siRNA but induced survivin (an anti-apoptotic protein) expression in colorectal and gastric tumors. In contrast, PCat-loaded with Survivin siRNA (PCat-siSurvivin) was not effective in vivo. A TPM/PCat-siSurvivin combination targeting Survivin overset the TPM-induced Survivin expression and intensified the anti-

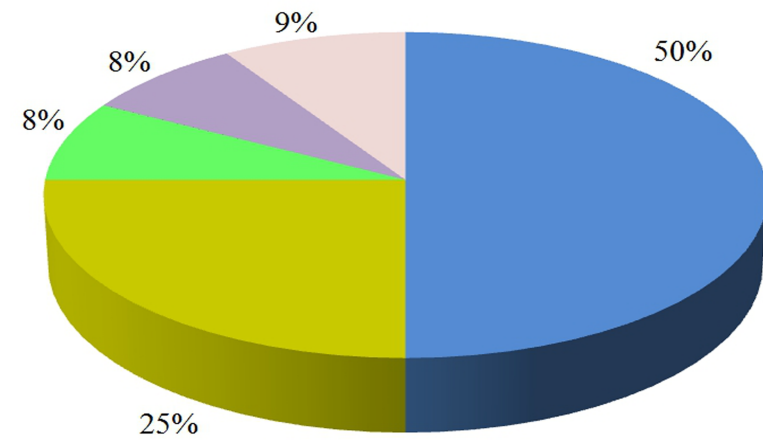

nanoparticle

- liqid\&liposome

$\square$ plasmid

virus

chemical modification
Fig. 1. Distribution of siRNA modification modes in tumors. 
tumor ability of TPM. The TPM/PCat-loaded with survivin siRNA (TPM/PCat-siSurvivin) combination could keep active in vivo only in this way (18). Another example was that Gli1-targeting siRNA reduced the expression of Gli1 and resulted in apoptosis and restrained pancreatic carcinoma growth (S38).

Others: Intratumor injection of adenovirus vector/siRNA targeting mouse II-17a gene (Ad-si-II-17A) resulted in a suppressive effect at tumor sites in terms of $I I-17 A$ levels as well as growth of the tumor, indicating that suppressing the expression of IL-17A played an important role in inhibiting tumor growth through stopping angiogenesis and $\mathrm{T}$ lymphocytes participating in the process (S39).

\section{Dermis}

A lentivirus vector and gelatin-conjugated siRNA were used in the treatment of dermis diseases. In a mouse model with xenografted psoriatic skin, local administration of lentivirus-encoded shRNAs targeting interleukin-12B (II-12B) resulted in reduced II-12B mRNA levels and meliorative skin (S46). To treat alopecia areata, siRNA conjugated with cationized gelatin microspheres was injected locally around hair follicle lesions in $\mathrm{C} 3 \mathrm{H} / \mathrm{HeJ}$ mice, offering new insights into understanding the potential application of siRNA-cationized gelatin microspheres (S47).

\begin{abstract}
Muscle
Adeno-associated virus serotype 6 (AAV6)-mediated mutant superoxide dismutase 1 ( $\operatorname{mSod} 1)$ was injected into the triceps surae muscle. After the silencing of mSod 1 in the innervating motor neurons (MNs), skeletal muscle was assessed. The data demonstrated the role of $m S o d 1$ in muscle atrophy alleviation and neuroprotection in an amyotrophic lateral sclerosis (ALS) mouse model (S48). With atelocollagen-mediated local injections of siRNA against Myostatin, significantly reduced Myostatin levels and increased skeletal muscle mass were found, confirming atelocollagen-mediated local injections of siRNA to be a potential therapeutic way to treat diseases like muscular atrophy (S49).
\end{abstract}

\section{Ear}

Naked siRNA has been used in ear disorder therapy. The first successful intra-tympanic injection was of naked NADPH oxidase 3 (Nox3)-siRNA applied into the antero-inferior region of the middle ear. Subsequently, Nox3 mRNA levels were reduced. This novel therapeutic may provide the options for patients suffering from cisplatin-induced hearing loss or other inner ear insults (S8).

\section{Joints}

Virus-mediated siRNA is the most commonly used form of local siRNA in joint tissues. The intervertebral disc has an avascular anatomical structure enclosed by the annulus fibrosis and end plate, making it possible to inject locally as a gene delivery site. BAFF (TNF superfamily member, B cell-activating factor) as a new gene silencing target has provided much potential in the treatment of rheumatoid arthritis (RA). After an intraarticular injection of lentivirus-shRNA-Baff into afflicted joint tissues, gene silencing of Baff was achieved, inflammation was suppressed, and autoimmune arthritis in the CIA model was improved (19). Adeno-associated virus type 5 (rAAV5) vectors encoding shRNA of Tnfa (tumor necrosis factor alpha) were injected into knee and ankle joints of mice with CIA. Lower inflammation levels in arthritis were achieved with the downregulated Tnfa gene levels (S50). Alexa Fluor 555-labeled Caspase3 siRNA was injected locally into the intervertebral discs of rabbits. Protein and mRNA levels of Caspase 3 were reduced, providing evidence for a role of Caspase 3 in intervertebral disc degeneration (S51).

\section{Heart}

There are few reports about local siRNA injection in the myocardium. A lower $\mathrm{Na}\left({ }^{+}\right) / \mathrm{H}^{+}$) exchanger 1 (Nhe1) level in mouse left ventricle after a siRNA-Nhe1 injection suggested a new technique for further exploration of genes in the cardiac area and the function of NHE1 (S11).

\section{CONCLUSIONS}

In this review, we summarized recently published data about the local application of siRNA. We found that local application of siRNA has been used successfully in some diseases models, such as brain diseases, eye diseases, and tumors. Eye and brain tissues are the 'hot' target tissues or organs for siRNA delivery. The key points of successful gene silencing in various organs rely on the design of effective siRNA sequences, selection of siRNA delivery routes, and formulation methods.

In the future, more tissues or organs, such as the oral cavity, nose, or vagina, may be targets for local siRNA application, and the formulations of siRNA may also need various changes. Nanoparticle-formulated siRNA may attract more attention due to the good biocompatibility, biodegradability, adsorbability, and low toxicity and immunogenicity. Considering the diseases involved, local application of siRNA may be a useful therapy method in the near future.

\section{ACKNOWLEDGEMENTS}

This work was supported by the National Natural Science Foundation of China (81271116, 81070819, and 31070835).

\section{REFERENCES}

1. Fire A, Xu S, Montgomery MK, Kostas SA, Driver SE and Mello CC (1998) Potent and specific genetic interference by double-stranded RNA in Caenorhabditis elegans. Nature 391, 806-811

2. Elbashir SM, Harborth J, Lendeckel W, Yalcin A, Weber K and Tuschl T (2001) Duplexes of 21-nucleotide RNAs mediate RNA interference in cultured mammalian cells. Nature 
$411,494-498$

3. Guo P, Coban O, Snead NM et al (2010) Engineering RNA for targeted siRNA delivery and medical application. Adv Drug Deliv Rev 62, 650-666

4. Lima WF, Prakash TP, Murray HM et al (2012) Single-stranded siRNAs activate RNAi in animals. Cell 150, 883-894

5. de Fougerolles AR (2008) Delivery vehicles for small interfering RNA in vivo. Hum Gene Ther 19, 125-132

6. Chen M, Gao S, Dong $M$ et al (2012) Chitosan/siRNA nanoparticles encapsulated in PLGA nanofibers for siRNA delivery. ACS Nano 6, 4835-4844

7. Pittella F, Cabral H, Maeda Y et al (2014) Systemic siRNA delivery to a spontaneous pancreatic tumor model in transgenic mice by PEGylated calcium phosphate hybrid micelles. J Control Release 178C, 18-24

8. Zimmermann TS, Lee AC, Akinc A et al (2006) RNAimediated gene silencing in non-human primates. Nature 441, 111-114

9. Ahmed Z, Kalinski H, Berry M et al (2011) Ocular neuroprotection by siRNA targeting caspase-2. Cell Death Dis 2, e173

10. Moreno-Montañés J, Sádaba B, Ruz V et al (2014) Phase I clinical trial of SYL040012, a small interfering RNA targeting $\beta$-adrenergic receptor 2 , for lowering intraocular pressure. Mol Ther 22, 226-232

11. Martínez T, González MV, Roehl I, Wright N, Pañeda C and Jiménez Al (2014) In vitro and in vivo efficacy of SYL040012, a novel siRNA compound for treatment of glaucoma. Mol Ther 22, 81-91

12. Yu D, Pendergraff $H$, Liu J et al (2012) Single-stranded
RNAs use RNAi to potently and allele-selectively inhibit mutant huntingtin expression. Cell 150, 895-908

13. DiFiglia M, Sena-Esteves M, Chase K et al (2007) Therapeutic silencing of mutant huntingtin with siRNA attenuates striatal and cortical neuropathology and behavioral deficits. Proc Natl Acad Sci U S A 104, 17204-17209

14. Dassie JP, Liu XY, Thomas GS et al (2009) Systemic administration of optimized aptamer-siRNA chimeras promotes regression of PSMA-expressing tumors. Nat Biotechnol 27, 839-849

15. Lai WY, Wang WY, Chang YC, Chang CJ, Yang PC and Peck K (2014) Synergistic inhibition of lung cancer cell invasion, tumor growth and angiogenesis using aptamersiRNA chimeras. Biomaterials 35, 2905-2914

16. Sakurai $Y$, Hatakeyama $H$, Sato $Y$ et al (2014) RNAi-mediated gene knockdown and anti-angiogenic therapy of RCCs using a cyclic RGD-modified liposomal-siRNA system. J Control Release 173, 110-118

17. Brock A, Krause S, Li H et al (2014) Silencing HoxA1 by intraductal injection of siRNA lipidoid nanoparticles prevents mammary tumor progression in mice. Sci Transl Med 6, 217ra2

18. Wang J, Lu Z, Yeung BZ, Wientjes MG, Cole DJ and Au JL (2014) Tumor priming enhances siRNA delivery and transfection in intraperitoneal tumors. J Control Release 178, 79-85

19. Lai Kwan Lam Q, King Hung Ko O, Zheng BJ and Lu L (2008) Local BAFF gene silencing suppresses Th17-cell generation and ameliorates autoimmune arthritis. Proc Natl Acad Sci U S A 105,14993-14998 